http://jmscr.igmpublication.org/home/

ISSN (e)-2347-176x ISSN (p) 2455-0450

crossref DOI: https://dx.doi.org/10.18535/jmscr/v7i11.98

Journal Of Medical Science And Clinical Research

\title{
Aberrant Bilateral Dual Renal Arteries with Accessory Arterial Supply to Left Kidney: Rare Variation: A Case Report
}

Authors

Dr Manpal Loona* M.B.B.S., D.N.B. CTVS, Dr Mohd. Javed Banday M.B.B.S., M.S.

Department of Cardiothoracic Vascular Surgery, ABVIMS \& Dr.R.M.L. Hospital, New Delhi

*Corresponding Author

Dr Manpal Loona

Department of Cardiothoracic Vascular Surgery, ABVIMS \& Dr.R.M.L. Hospital, New Delhi, India

\begin{abstract}
Usually renal arteries arise from lateral or anterolateral aspect with single supply to each kidney. Renal vasculature may vary during embryological development. These variations have been termed accessory, unilateral or bilateral dual renal arteries. These renal variations provide additional information for interventional radiologists, vascular and transplant surgeons and urologist to understand better anatomy and outcomes in day to day practice.

Keywords: dual renal artery, aberrant renal artery, bilateral accessory renal arteries.
\end{abstract}

\section{Introduction}

Renal artery originates from anterolateral or lateral aspect of abdominal aorta right at level of $\mathrm{L} 1$, as direct aortic branches, below the origin of superior mesenteric artery. Normally, kidney receives single renal artery and origin of right sided renal artery is seen longer and slightly higher than left. On either side, renal artery enters the hilum, divides into anterior and posterior branches, thereafter into segmental arteries ${ }^{[1]}$.

Renal artery variations are not uncommon. Renal artery variations include accessory renal artery, unilateral or bilateral dual renal arteries. These variations are related to embryological development of kidney with its defects from lateral mesonephric branches of dorsal aorta and its arterial supply during ascend to the lumbar region. During development, both kidneys ascend to lumbar region and their arterial supply also shifts from common iliac arteries to the abdominal aorta $^{[2]}$. An additional renal artery arises from the abdominal aorta or common iliac vessels either above or below the main renal artery. Objective of this case report is addition to the known variations of blood supply of kidney ${ }^{[3]}$.

\section{Case Report}

We hereby report a case of young man 32 year old, road traffic accident presented in emergency with multiple abrasions with acute abdominal pain. On evaluation patient was hemodynamically stable with tenderness in right pelvic region. Rest of clinical examination was unremarkable. Urgent abdominal ultrasound was done which revealed no significant findings. Routine blood investigations were done including liver function test, renal function test and pancreatic enzymes. CT contrast abdominal aortography was done as intestinal 
ischemia was suspected clinically, in view of persistent abdominal pain. CT revealed no significant changes in arterial supply to major abdominal vessels. Along with this we encountered incidental abnormal renal arterial supply to both the renal arteries with additional accessory to left renal artery. We found that right kidney had dual supply with both superior lobe and inferior lobe having its separate supply with no supply through hilum. Whereas on the left side dual renal arteries were found supplying superior and inferior lobes through superior and inferior branches dividing just before entering to the hilum along with accessory branch to left renal arteries [Figure. $1 \& 2$ ].

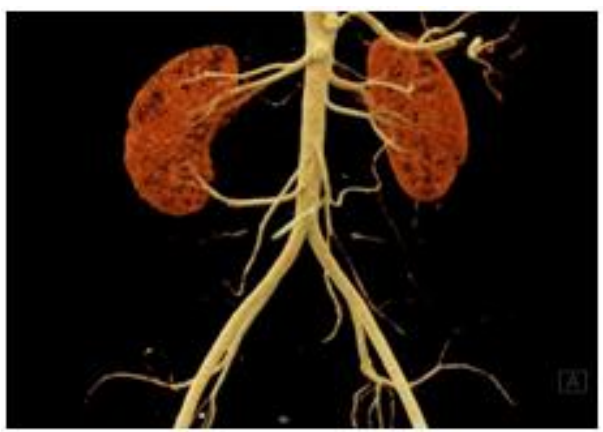

Figure.1: Depicting 3D imaging of right kidney with upper and lower lobes with dual arterial supply with no hilar branch, whereas left renal artery with dual hilar arteries and accessory branch supplying left kidney.

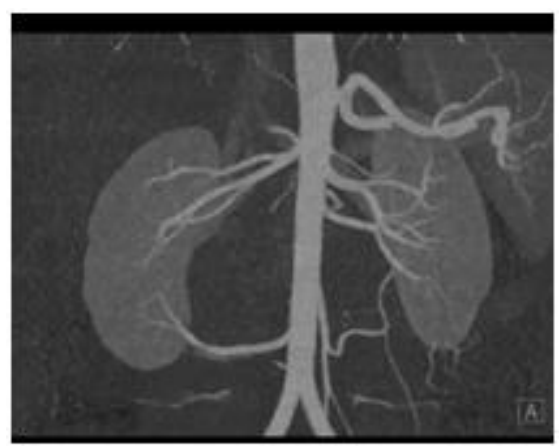

Figure. 2: showing CT Aortogram with aberrant vascular supply to both kidneys.

On contemporary, patient was kept in ICU for one day, as his pain subsided with analgesics and symptoms improved. Thereafter, patient was shifted to ward and was discharged on 3rd day with follow up after week with no complaints.

\section{Discussion}

Usual origin of renal arteries arises from lateral or anterolateral aspect of abdominal aorta at L1 level. Development of renal arteries as suggested that the primordial kidneys lie close to each other in the pelvis and ventral to the sacrum. As the foetus grows, the kidneys gradually come to lie in the abdomen and move apart gradually. During embryological changes both kidneys receive their blood supply from vessels that lie close to them, with gradual changes observed, as in origin initially from common iliac arteries, then from distal end of aorta followed by separate permanent new branches at term. Usually, the caudal renal vessels undergo involution and disappear as foetus grows. These caudal renal arteries sometimes doesn't involute and tend to become accessory vasculature to kidneys. Among renal vascular variations, Accessory renal arteries are the most common variant followed by unilateral and bilateral multiple renal arteries. Mostly Accessory renal arteries are found arising from the aorta or iliac arteries and very rarely from lower thoracic aorta or from lumbar or mesenteric arteries at the level between $\mathrm{T} 11$ to $\mathrm{L} 4$ vertebra $^{[1-4]}$. There are very few cases being reported over the time with its variation of renal vascular anomalies

Bordei et al found bilateral double renal artery with incidence of $2.2 \%$ in a study of 272 kidneys ${ }^{[5]}$. Bergman et al reported a case in their literature about right kidney having two renal arteries, and double testicular arteries ${ }^{[6]}$. K S Satyapal et al also documented in their study in 130 angiogram and 32 cadavers, and mentioned $23 \%$ of double renal artery on right side, $32 \%$ on left side and $10 \%$ cases bilaterally ${ }^{[7]}$. Albohassan et al found incidence of bilateral double renal artery is less than $1 \%$ in angiography as in renal transplant donor ${ }^{[8]}$.

Rusu et al. mentioned in his reported case, bilateral double renal arteries with its branching pattern as superior and inferior renal arteries with their origin from the abdominal aorta ${ }^{[9]}$. Bayramoglu et al. reported bilateral accessory renal arteries originating from the abdominal aorta 
with an additional right renal vein accompanying the right renal artery ${ }^{[10]}$. Bulic et al. reported a case with two hilar renal arteries originating from the aorta on right side and left kidney having three arteries originating from the aorta, one at hilar position and two emerging at its upper and lower poles $^{[11]}$. Gupta et.al reported a case with right kidney receiving three renal arteries and draining by two renal veins. The left kidney was found receiving two renal arteries ${ }^{[12]}$. Prajkta AT et al mentioned in their reported case having right side the accessory renal artery originating from the abdominal aorta just above the main renal artery and left side the accessory renal artery originating from the abdominal aorta about $1 \mathrm{~cm}$ above the main renal artery ${ }^{[13]}$. Ranjana Singh Arya et al also reported similar cases having bilateral accessory renal arteries are found originating from the abdominal aorta. The right accessory renal artery is originating higher than the left accessory renal artery ${ }^{[14]}$.

Therefore the knowledge of renal vasculature remains important for renal transplant, radiologists, vascular surgeons, and as anatomical variations. We hereby report a case of aberrant bilateral dual renal arteries with accessory arterial supply to left kidney with its variable branching pattern origin from Aorta.

\section{Conclusion}

These aberrant variations of bilateral renal vessels provides knowledge to prevent complications during renal surgeries, especially in treatment of renal trauma, renal transplantation, renal artery embolization, surgery for abdominal aortic aneurysm and conservative or radical renal surgeries and radiological renal interventions.

\section{References}

1. Standring S. Gray's Anatomy. [2005]: The Anatomical Basis of Clinical Practice. 39th Ed. London, Elseiver Churchill Livingstone Publishers.; 1274-1275.
2. Moore KL, Arthur DF, Anne MR. [2010]: Clinically Oriented Anatomy. 6th Ed. New Delhi: Wolters Kluwer; p. 298.

3. Moore KL, Persaud TV. [2008]: The Developing Human. Clinically Oriented Embryology. 8th Ed. New Delhi: Elsevier; p. 249-50.

4. Nasir Syed Mir et al; [2008]: Bilateral Duplication of Renal Vessels: Anatomical, Medical and Surgical perspective: International Journal of Health Sciences Vol. 2 No. 2 july(Jumad'aThani 1429 H).

5. Bordei P, Sapte E, Iliescu D. [2004]: Double renal arteries originating from the aorta. SurgRadiolAnat; 26:474-9.

6. Bergman RA, Cassell MD, Sahinoglu K, Heidger PM Jr. [1992]: Human doubled renal and testicular arteries. Ann Anat; 174: 313-315.

7. Satyapal KS, Haffejee AA, Sing B, Ramsaroop L, Robbs JV, Kaliden JM. [2001]: Additional renal arteries: Incidence and morphometry. SurgRadiol Anat.; 23(1): 33-38.

8. Alboo Hssan, Shakeri et al, [2007]: Bipolar supernumerary renal artery: surgical and radiological anatomy, Anatomic variation; Vol 29, 89-92.

9. Rusu MC. [2006]: Human bilateral doubled renal and testicular arteries with a left testicular arterial arch around the left renal vein. Rom J MorpholEmbryol; 47: 197-200

10. Bayramoglu A, Demiryurek D, Erbil KM. [2003]: Bilateral additional renal arteries and an additional right renal vein associated with unrotated kidneys. Saudi Med J.; 24: 535-537.

11. Bulic K, Ivkic G, Pavic T. [1996]: A case of duplicated right renal artery and triplicated left renal artery. Ann Anat.; 178: 281-283.

12. Gupta, Vanita, Sheetal Kotgirwar, Soumitra Trivedi, Rashmi Deopujari, and Vikrant Singh.[2010]: Bilateral variation 
in renal vasculature. International Journal of Anatomical Variations; 3: 53-55.

13. Prajkta A Thete, Mehera Bhoir, M.V. Ambiye [2014]: Bilateral duplication of renal arteries: case report. Int J Anat Res; 2(3): 580- 582.

14. Arya RS, Kumar A, Jangde S, Saha K, Tayade SP. [2015]: Presence of Bilateral Accessory Renal Arteries: A Rare Case Report. Int J Sci Stud; 3(3):119-121. 